\title{
Etude comparée des paramètres agromorphologiques de dix (10) variétés de patate douce (Ipomoea batatas (L.) Lam cultivées au champ dans deux (2) conditions climatiques au Tchad et au Burkina Faso
}

\author{
Alain Ignassou DJINET*, Rasmata NANA, Zoumbiéssé TAMINI et Badoua BADIEL
}

Laboratoire de Biosciences, Université de Ouagadougou, 03 BP 7021 Ouagadougou 03, Burkina Faso.

*Auteur correspondant,E-mail : alain_djinet@yahoo.fr

\section{RESUME}

Cette étude a été réalisée dans le but d'évaluer les paramètres agromorphologiques des variétés de patate douce cultivée à Bongor au Tchad et à Ouagadougou au Burkina Faso. La longueur du tubercule, le diamètre du tubercule, le nombre du tubercule par plant, le rendement, le poids moyen du tubercule, le rapport diamètre/longueur du tubercule et la matière sèche du tubercule ont été évalués. Les résultats obtenus ont montré que la variété BF108 s'est distinguée des autres dans les deux conditions climatiques en termes de rendement, longueur et diamètre du tubercule, de poids moyen du tubercule, de rapport diamètre/longueur du tubercule et de matière sèche de la biomasse aérienne. La variété BF 11 de Ouagadougou a le nombre des tubercules par plant le plus élevé. Tandis qu'à Bongor, la variété BF 108 a ce nombre élevé. A Bongor (Tchad) tout comme à Ouagadougou (Burkina Faso) la variété BF 140 a exprimé une teneur importante en matière sèche du tubercule.

() 2015 International Formulae Group. All rights reserved.

Mots clés : paramètres agronomiques, conditions climatiques, patate douce.

\section{INTRODUCTION}

La patate douce (Ipomoea batatas (L.) Lam est l'un des plus importants aliments glucidiques consommés en Afrique sub-saharienne. Elle s'adapte bien aux sols relativement pauvres et des précipitations irrégulières Mbanaso et al.(2012). Parmi les espèces du genre Ipomoea, elle est la seule à produire des racines tubéreuses comestibles (Afuape et al., 2014). Mais, les rendements moyens en Afrique sont faibles et instables par rapport aux chiffres que l'on peut obtenir dans des conditions optimales (Bell et al., 2000). Les contraintes de production les plus fréquentes sont: les aléas climatiques, les conditions édaphiques, les techniques de production et de conservation rudimentaires, manque de variétés à rendement élevé et stable, attaque des charançons au champ et en stock, possibilité de commercialisation limitées, non valorisation des sous produits et manque de disponibilité de bonnes semences en temps utile. Pour pallier à cette situation, il est donc nécessaire de mettre à la disposition des agriculteurs des variétés d'intérêt capital. L'objectif de ce travail est d'évaluer les paramètres agromorphologiques des variétés 
de patate douce dans les deux conditions climatiques afin de fournir des éléments nécessaires pour l'amélioration variétale.

\section{MATERIEL ET METHODES Matériel Matériel végétal}

Les boutures de patate douce qui ont fait l'objet de cette étude proviennent de la station de l'Institut National de l'Environnement et de la Recherche Agricole (INERA) de Kamboinsé (Burkina Faso). Dix (10) variétés ont servi de matériel de base. Il s'agit des variétés $B F$ 139, Jewel, BF 13, BF 11, TIB440060, BF 59, BF 142, BF 108, BF 140 et BF 40. Les caractéristiques des différentes variétés sont consignées dans le Tableau 1

\section{Site expérimental}

Les expérimentations ont été conduites du 20 juillet au 10 novembre 2013 soit une durée de 102 jours, dans le jardin expérimental de l'Ecole Normale Supérieure de Bongor (10.280 ${ }^{\circ}$ latitude Nord et à $15.370^{\circ}$ longitude Est) au Tchad et du 12 juin au 25 septembre 2012 soit une durée de 106 jours, dans le jardin expérimental de l'Unité de Formation et de la Recherche en Sciences de la Vie et de la Terre situé dans l'enceinte de l'Université de Ouagadougou $\left(1^{\circ} 31\right.$ longitude Ouest et $12^{\circ} 21$ latitude Nord). Les précipitations moyennes enregistrées pendant la période culturale étaient de 227,6 $\mathrm{mm}$ dans les conditions climatiques de Ouagadougou et de 178,49 mm dans les conditions de Bongor. Dans la même période, la température moyenne était de $28,1^{\circ} \mathrm{C}$ à Ouagadougou et de $33,1^{\circ} \mathrm{C}$ à Bongor. Cependant, l'humidité moyenne était de 72 $\%$ à Ouagadougou et 74,99\% à Bongor. Les sols utilisés étaient sablo-limoneux mais dans les conditions climatiques de Ouagadougou, le pourcentage d'argile et de limons a été beaucoup plus important.

\section{Méthodes}

Dispositif expérimental

L'essai était conduit sur les deux sites durant les saisons des pluies de 2012 et 2013 , dans un dispositif expérimental en blocs randomisés en 3 répétitions. Trois (3) billons ont été utilisés pour chaque variété. Pour chaque variété, 4 boutures ont été retenues soit 360 boutures pour les dix (10) variétés. La distance entre les plants d'une même variété sur le billon a été de $50 \mathrm{~cm}$ et l'écartement entre les billons a été de $1 \mathrm{~m}$. La densité à l'hectare a été de 20000 plants.

\section{Collecte de données}

Le poids et le nombre du tubercule par plant pour chaque variété ont été relevés. La longueur et le diamètre du tubercule ont été mesurés. Pour cela, la règle graduée et le pied à coulisse ont été respectivement utilisés. Pour le poids du tubercule, la balance a été utilisée. Pour obtenir la matière sèche, trois (3) tubercules de chaque variété pris au hasard dans les blocs ont été épluchés et découpés à la main en fines lamelles puis mélangés. Pour chaque variété trois (3) échantillons de $10 \mathrm{~g}$ chacun ont été séchés à l'étuve à $105{ }^{\circ} \mathrm{C}$ pendant 24 heures jusqu'à l'obtention d'un poids constant. Le pourcentage de la matière sèche a été calculé par la formule suivante : Taux d'humidité : \% $\mathrm{H}_{2} \mathrm{O}=\left(\left(\mathrm{M}_{1}-\mathrm{M}_{2}\right) /\left(\mathrm{M}_{1}{ }^{-}\right.\right.$ $\left.\left.\mathrm{M}_{0}\right)\right) \times 100 \% \mathrm{MS}=100-\% \mathrm{H}_{2} \mathrm{O}$ $\mathrm{M}_{0}$ : poids du creuset à vide $\mathrm{M}_{1}$ : poids du creuset contenant le materiel à secher.

$\mathrm{M}_{2}$ : poids de l'ensemble creuset et produit après étuvage

MS: matière séche.

La matière sèche de la biomasse aérienne a été déterminée par pesée de la tige et des feuilles; le poids frais obtenu est noté $\mathrm{P}_{1}$, puis la partie aérienne (tige et feuilles) a été mise à l'étuve à $105^{\circ}$ pendant 72 heures. A la sortie de l'étude, une série de pesées a été effectuée jusqu'à l'obtention du poids constant, noté poids $\mathrm{P}_{2}$. La différence de 
poids noté $\Delta \mathrm{P}$ représente la matière sèche de la biomasse aérienne.

\section{Traitement et analyse statistiques de données.}

Les résultats relatifs aux paramètres évalués ont été soumis à une analyse de variance (ANOVA). Les tests de comparaison des moyennes ont été effectués selon la méthode de Fisher au seuil de 5\%. Le logiciel XLSAT version 7.5.2 a été utilisé à cet effet.

\section{RESULTATS}

Les Tableaux 2 et 3 présentent respectivement les résultats des analyses statistiques des expérimentations réalisées dans les conditions climatiques de Bongor et de Ouagadougou en saison des pluies. Concernant la longueur du tubercule, il existe une différence significative $(\mathrm{P}<$ 0,0001) entre les variétés dans les deux conditions climatiques. Cette longueur a varié de 6,82 à 20,30 cm à Bongor. La valeur la plus élevée est notée chez la variété $\mathrm{BF} 40(20,30 \mathrm{~cm})$ et la plus faible longueur notée aussi chez la variété BF 140 $(6,82 \mathrm{~cm})$. Par contre à Ouagadougou, elle a varié de 10,50 à $16,55 \mathrm{~cm}$. La variété $B F$ 11 a les tubercules les plus longs (16,55 $\mathrm{cm})$ et les tubercules les plus courts ont été notés chez la variété BF $140(10,50 \mathrm{~cm})$. Pour le diamètre du tubercule il existe également une différence hautement significative $(\mathrm{P}<0,0001)$ entre les variétés cultivées à Bongor et une différence significative $(\mathrm{P}<0,0001)$ entre les variétés cultivées dans les conditions climatiques de Ouagadougou. A Bongor, le diamètre du tubercule a varié de 2,38 à 9,41 cm. La variété dont les tubercules possèdent un diamètre le plus grand est la BF $108(9,41$ $\mathrm{cm})$ et celle dont les tubercules possèdent le plus petit diamètre est la BF $140(2,38$ $\mathrm{cm})$. Tandis qu'à Ouagadougou, le diamètre du tubercule a varié de 3,42 à
$9,86 \mathrm{~cm}$. La variété BF 108 a les tubercules les plus gros avec en moyenne $9,86 \mathrm{~cm}$ de diamètre. La variété qui a les tubercules les plus petits en diamètre est la variété BF 140 (3,42 cm en moyenne).

Quant au nombre du tubercule par plant, il a varié de 1 à 4 à Bongor et de 2 à 5 à Ouagadougou. Entre les variétés, une différence significative $(\mathrm{P}<0,0001)$ a été observée à Bongor tout comme à Ouagadougou. A Bongor la valeur la plus élevée est observée chez les variétés BF 108, BF 40, BF 11, BF 13 et Jewel soit 4 tubercules par plant et la plus faible valeur est notée chez la variété BF 140 soit un tubercule par plant. Par contre à Ouagadougou, le plus grand nombre est noté chez les variétés BF 108, BF 59 et BF 11 soit 5 tubercules par plant et le plus petit nombre est aussi noté chez la variété BF 139 soit 2 tubercules par plant.

Le rendement en tubercules à la récolte a varié entre 0,94 et 45,15 t/ha à Bongor et de 2,23 à 24,02 t/ha à Ouagadougou. A Bongor, le rendement le plus élevé est enregistré chez la variété $\mathrm{BF} 108(45,15 \mathrm{t} / \mathrm{ha})$ et le rendement le plus faible est obtenu chez la variété $\mathrm{BF}$ 140 (0,94 t/ha). A Ouagadougou, la variété BF 108 a également un rendement plus grand soit 24,02 t/ha et la variété BF 140 a un rendement beaucoup plus faible soit 2,23 t/ha. Une différence hautement significative $(\mathrm{P}<$ 0,0001) a été notée entre les variétés dans les $\operatorname{deux}(2)$ conditions climatiques.

Le poids moyen du tubercule a varié d'une variété à une autre, entre 47,11 et $572,19 \mathrm{~g}$ à Bongor. A Ouagadougou, le poids moyen du tubercule était compris entre 37,9 et 241,46 g. A Bongor, les tubercules de la variété $\mathrm{BF} 108$ ont le poids le plus élevé soit $572,19 \mathrm{~g}$ et le poids moyen du tubercule le plus faible est noté chez la variété BF 140 $(47,11$ g). A Ouagadougou, la variété qui a présenté des tubercules plus lourds est la variété BF 108 (241,46 g) et les tubercules les plus légers sont obtenus chez la variété $\mathrm{BF}$ $140(37,9 \mathrm{~g})$. 
Tableau 1: Caractéristiques des variétés étudiées.

\begin{tabular}{|c|c|c|c|c|c|c|c|c|c|c|}
\hline \multirow{2}{*}{ Caractéristiques } & \multicolumn{10}{|c|}{ Variétés } \\
\hline & BF 139 & JEWEL & BF 13 & BF 11 & TIB-440060 & BF 59 & BF 142 & BF 108 & BF 140 & BF 40 \\
\hline Origine & $\begin{array}{l}\text { Collection } \\
\text { Burkina }\end{array}$ & Pérou & $\begin{array}{l}\text { Collection } \\
\text { Burkina }\end{array}$ & $\begin{array}{c}\text { Collection } \\
\text { Burkina }\end{array}$ & Pérou & $\begin{array}{l}\text { Collection } \\
\text { Burkina }\end{array}$ & $\begin{array}{l}\text { Collection } \\
\text { Burkina }\end{array}$ & $\begin{array}{l}\text { Collection } \\
\text { Burkina }\end{array}$ & $\begin{array}{l}\text { Collection } \\
\text { Burkina }\end{array}$ & $\begin{array}{l}\text { Collection } \\
\text { Burkina }\end{array}$ \\
\hline Durée du cycle & 3 mois & 3-4 mois & 3 mois & 3 mois & 3-4 mois & 3 mois & 3 mois & 3 mois & 3 mois & 3 mois \\
\hline Type de plante & déployé & déployé & Très déployé & Déployé & déployé & Très déployé & Semi érigé & Très déployé & Déployé & $\begin{array}{c}\text { Très } \\
\text { déployé }\end{array}$ \\
\hline $\begin{array}{l}\text { Aspect général du } \\
\text { limbe foliaire }\end{array}$ & Triangulaire & triangulaire & Triangulaire & $\begin{array}{l}\text { Triangulai } \\
\text { re }\end{array}$ & hasté & triangulaire & $\begin{array}{c}\text { triangulair } \\
\mathrm{e}\end{array}$ & Triangulaire & Hasté & Triangulaire \\
\hline $\begin{array}{l}\text { Nombre de lobes } \\
\text { des feuilles }\end{array}$ & 5 & 7 & 3 & 5 & 5 & 9 & 1 & 3 & 5 & 9 \\
\hline $\begin{array}{l}\text { Forme de la racine } \\
\text { tubéreuse }\end{array}$ & $\begin{array}{l}\text { Longue et } \\
\text { elliptique }\end{array}$ & $\begin{array}{l}\text { Longue et } \\
\text { irrégulière }\end{array}$ & Elliptique & $\begin{array}{l}\text { Longue et } \\
\text { oblongue }\end{array}$ & Elliptique & $\begin{array}{l}\text { Longue et } \\
\text { elliptique }\end{array}$ & $\begin{array}{l}\text { Ronde } \\
\text { elliptique }\end{array}$ & $\begin{array}{l}\text { Longue et } \\
\text { elliptique }\end{array}$ & Dispersée & dispersée \\
\hline Couleur de la peau & orange & orange & Blanche & Rouge & Orange & crème & Rose & rouge & Crème & rouge \\
\hline Couleur de la pulpe & orange & Orange foncé & Jaune pâle & Jaune pâle & orange & Jaune pâle & blanche & blanche & blanche & Jaune pâle \\
\hline
\end{tabular}


A. I. DJINET et al. / Int. J. Biol. Chem. Sci. 9(3): 1243-1251, 2015

Tableau 2 : Paramètres agronomiques mesurés à Bongor au Tchad en saison des pluies.

\begin{tabular}{|c|c|c|c|c|c|c|c|c|c|c|c|c|}
\hline & $\begin{array}{c}\text { F de } \\
\text { Fisher }\end{array}$ & $\begin{array}{c}P \text { au seuil de } \\
5 \%\end{array}$ & BF 139 & Jewel & BF 13 & BF 11 & $\begin{array}{c}\text { TIB- } \\
440060\end{array}$ & BF 59 & BF 142 & BF 108 & BF 140 & BF 40 \\
\hline $\mathrm{LgT}$ & 29,41 & $<0,0001^{*}$ & $13,78^{\mathrm{cd}}$ & $15,94^{\mathrm{bc}}$ & $15,71^{\mathrm{bc}}$ & $22,06^{\mathrm{a}}$ & $11,94^{\mathrm{d}}$ & $16,71^{b}$ & $11,89^{\mathrm{d}}$ & $15,30^{\mathrm{bc}}$ & $6,82^{\mathrm{e}}$ & $20,30^{\mathrm{a}}$ \\
\hline DmT & 16,28 & $<0,0001^{*}$ & $6,68^{\mathrm{b}}$ & $6,23^{\mathrm{b}}$ & $5,79^{\mathrm{b}}$ & $6,74^{\mathrm{b}}$ & $6,17^{\mathrm{b}}$ & $9,29^{\mathrm{a}}$ & $6,28^{\mathrm{b}}$ & $9,41^{\mathrm{a}}$ & $2,38^{\mathrm{c}}$ & $6,04^{\mathrm{b}}$ \\
\hline NbT/plant & 2,74 & $=0,028$ & $3,00^{\mathrm{ab}}$ & $4,00^{\mathrm{ab}}$ & $4,00^{\mathrm{ab}}$ & $4,00^{\mathrm{a}}$ & $3,00^{\mathrm{ab}}$ & $3,00^{\mathrm{ab}}$ & $2,00^{\mathrm{bc}}$ & $4,00^{\mathrm{a}}$ & $1,00^{\mathrm{c}}$ & $4,00^{\mathrm{a}}$ \\
\hline $\mathrm{RdT}$ & 14,46 & $<0,0001^{*}$ & $15,93^{\mathrm{cd}}$ & $16,85^{\mathrm{cd}}$ & $16,87^{\mathrm{cd}}$ & $41,07^{\mathrm{ab}}$ & $11,026^{\text {cde }}$ & $33,83^{b}$ & $6,82^{\mathrm{de}}$ & $45,15^{\mathrm{a}}$ & $0,94^{\mathrm{e}}$ & $20,50^{\mathrm{b}}$ \\
\hline PMT & 45,84 & $<0,0001^{*}$ & $279,52^{\mathrm{b}}$ & $210,78^{b c}$ & $214,28^{\mathrm{bc}}$ & $543,78^{a}$ & $205,72^{\mathrm{bc}}$ & $566,23^{\mathrm{a}}$ & $152,74^{\mathrm{c}}$ & $572,19^{\mathrm{a}}$ & $47,11^{\mathrm{d}}$ & $259,40^{\mathrm{b}}$ \\
\hline $\mathrm{DmT} / \mathrm{LgT}$ & 9,24 & $<0,0001^{*}$ & $0,48^{\mathrm{bc}}$ & $0,38^{\mathrm{cd}}$ & $0,36^{\mathrm{d}}$ & $0,30^{\mathrm{d}}$ & $0,51^{\mathrm{ab}}$ & $0,56^{\mathrm{ab}}$ & $0,53^{\mathrm{ab}}$ & $0,61^{\mathrm{a}}$ & $0,36^{\mathrm{d}}$ & $0,29^{\mathrm{d}}$ \\
\hline MST & 58,59 & $<0,0001^{*}$ & $27,17^{\mathrm{de}}$ & $23,40^{\mathrm{fg}}$ & $25,53^{\mathrm{ef}}$ & $21,41^{\mathrm{g}}$ & $31,93^{b c}$ & $21,70^{\mathrm{g}}$ & $25,93^{\mathrm{ef}}$ & $34,26^{\mathrm{b}}$ & $45,23^{\mathrm{a}}$ & $29,60^{\text {cd }}$ \\
\hline BMA & 503,39 & $<0,0001^{* * * *}$ & $55,30^{\mathrm{g}}$ & $78,76^{\mathrm{g}}$ & $1677,82^{\mathrm{b}}$ & $1401,04^{\mathrm{cd}}$ & $816,04^{\mathrm{f}}$ & $1383,70^{\mathrm{d}}$ & $51,76^{\mathrm{g}}$ & $2973,03^{\mathrm{a}}$ & $9,20^{\mathrm{g}}$ & $1231,81^{\mathrm{e}}$ \\
\hline
\end{tabular}

tubercule ; NbT/plant : nombre du tubercule par plant ; RdT : rendement en tubercules ; PMT : poids moyen du tubercule ; DmT/LgT : rapport diamètre et longueur du tubercule ; MST : matière sèche du tubercule ; BMA : biomasse aérienne.

Tableau 3 : Paramètres agronomiques mesurés à Ouagadougou au Burkina Faso en saison des pluies.

\begin{tabular}{|c|c|c|c|c|c|c|c|c|c|c|c|c|}
\hline & $\begin{array}{c}\text { F de } \\
\text { Fisher }\end{array}$ & $\begin{array}{c}P \text { au seuil de } \\
5 \%\end{array}$ & BF 139 & Jewel & BF 13 & BF 11 & $\begin{array}{c}\text { TIB- } \\
440060\end{array}$ & BF 59 & BF 142 & BF 108 & BF 140 & BF 40 \\
\hline LgT & 17,88 & $<0,0001^{* * *}$ & $13,36^{\mathrm{d}}$ & $13,74^{\mathrm{cd}}$ & $15,61^{\mathrm{abc}}$ & $16,55^{\mathrm{a}}$ & $10,25^{\mathrm{e}}$ & $15,00^{\mathrm{bc}}$ & $12,44^{\mathrm{d}}$ & $16,34^{\mathrm{a}}$ & $10,50^{\mathrm{e}}$ & $13,25^{\mathrm{d}}$ \\
\hline DmT & 46,18 & $<0,0001^{*}$ & $4,60^{f}$ & $5,20^{\mathrm{ef}}$ & $6,79^{\mathrm{bc}}$ & $6,84^{\mathrm{bc}}$ & $3,58^{\mathrm{g}}$ & $7,03^{\mathrm{b}}$ & $5,72^{\mathrm{de}}$ & $9,86^{\mathrm{a}}$ & $3,42^{\mathrm{g}}$ & $6,10^{\mathrm{cd}}$ \\
\hline NbT/plant & 2,11 & 0,078 & $2,00^{\mathrm{b}}$ & $4,00^{\mathrm{ab}}$ & $4,00^{\mathrm{ab}}$ & $5,00^{\mathrm{a}}$ & $4,00^{\mathrm{ab}}$ & $5,00^{\mathrm{a}}$ & $4,00^{\mathrm{ab}}$ & $5,00^{\mathrm{a}}$ & $3,00^{\mathrm{ab}}$ & $3,00^{\mathrm{ab}}$ \\
\hline RdT & 10,02 & $<0,0001^{*}$ & $4,64^{\mathrm{ef}}$ & $9,07^{\mathrm{de}}$ & $14,73^{\mathrm{bcd}}$ & $15,56^{\mathrm{bc}}$ & $4,67^{\mathrm{ef}}$ & $18,68^{\mathrm{ab}}$ & $9,41^{\text {cde }}$ & $24,02^{\mathrm{a}}$ & $2,23^{\mathrm{f}}$ & $11,72^{\text {cd }}$ \\
\hline PMT & 807,58 & $<0,0001^{*}$ & $116,01^{\mathrm{e}}$ & $113,11^{\mathrm{e}}$ & $183,47^{\mathrm{c}}$ & $155,68^{\mathrm{d}}$ & $57,95^{\mathrm{f}}$ & $187,12^{\mathrm{c}}$ & $117,11^{\mathrm{e}}$ & $241,46^{\mathrm{a}}$ & $37,29^{\mathrm{g}}$ & $195,56^{\mathrm{b}}$ \\
\hline DmT/LgT & 8,95 & $<0,0001^{*}$ & $0,34^{\mathrm{cd}}$ & $0,38 \mathrm{~cd}$ & $0,43^{\mathrm{bc}}$ & $0,42^{\mathrm{bc}}$ & $0,35^{\mathrm{cd}}$ & $0,47^{\mathrm{b}}$ & $0,46^{\mathrm{b}}$ & $0,60^{\mathrm{a}}$ & $0,33^{\mathrm{d}}$ & $0,46^{\mathrm{b}}$ \\
\hline MST & 31,32 & $<0,0001^{*}$ & $30,89^{\mathrm{b}}$ & $28,70^{\mathrm{bc}}$ & $25,77^{\text {bcdef }}$ & $21,29^{\mathrm{ef}}$ & $29,33^{\mathrm{b}}$ & $20,49^{\mathrm{f}}$ & $24,16^{\text {cdef }}$ & $27,87^{\text {bcd }}$ & $53,89^{\mathrm{a}}$ & $23,62^{\text {def }}$ \\
\hline BMA & 89233,64 & $<0,000^{*}$ & $24,52^{\mathrm{i}}$ & $57,52^{\mathrm{h}}$ & $1060,37^{\mathrm{c}}$ & $1108,93^{\mathrm{b}}$ & $379,49^{f}$ & $657,95^{\mathrm{e}}$ & $94,43^{\mathrm{g}}$ & $2091,36^{\mathrm{a}}$ & $6,26^{\mathrm{J}}$ & $935,92^{\mathrm{d}}$ \\
\hline
\end{tabular}

du tubercule ; NbT/plant : nombre du tubercule par plant ; RdT : rendement en tubercules ; PMT : poids moyen du tubercule ; DmT/LgT : rapport diamètre et longueur du tubercule ; MST : matière sèche du tubercule ; BMA : biomasse aérienne. 
Il existe une différence hautement significative $(\mathrm{P}<0,0001)$ entre les variétés pour le poids moyen du tubercule dans les deux (2) conditions climatiques. Le rapport diamètre/longueur du tubercule a varié de 0,30 à 0,61 pour les variétés cultivées à Bongor et de 0,33 à 0,60 pour les variétés cultivées à Ouagadougou. Le rapport le plus important enregistré à Bongor est observé chez la variété BF $108(0,61)$ et le plus faible est noté chez la variété BF 11(0,30). A Ouagadougou, le rapport le plus grand est trouvé aussi chez la variété BF $108(0,60)$ et le plus faible est noté chez la variété $\mathrm{BF}$ 140(0,33). Une différence significative $(\mathrm{P}<$ $0,0001)$ a été notée entre les variétés dans les $\operatorname{deux}(2)$ conditions climatiques. Mais les valeurs du rapport obtenues dans les deux conditions ne sont pas significativement différentes.

La matière sèche du tubercule a varié de 21,41 à 45,23\% à Bongor et de 21,29 à $53,89 \%$ à Ouagadougou. A Bongor la variété qui a la matière sèche la plus élevé est la BF $140(45,23 \%)$ et celle qui a la plus faible teneur en matière sèche est la $\mathrm{BF} 11(21,41$ $\%)$. A Ouagadougou, la variété BF 140 a également une teneur en matière sèche élevée soit $53,89 \%$ et la plus faible teneur est aussi notée chez la variété BF 11 soit 21,29\%. Une différence significative $(\mathrm{P}<0,0001)$ a été notée entre les variétés pour les deux conditions climatiques. La matière sèche de la biomasse aérienne a varié de 9,20 à 2973,03 g à Bongor et de 6,26 à 2091 g à Ouagadougou. La plus faible valeur obtenue à Bongor est enregistrée chez la variété BF 140 $(9,20 \mathrm{~g})$ et la plus grande valeur est obtenue chez la variété BF $108 \quad(2973,03$ g). A Ouagadougou, la matière sèche de la biomasse aérienne la plus forte est enregistrée chez la variété BF 108 (2091 g) et la plus faible matière est notée chez la variété $\mathrm{BF} 140(6,29$ g). Entre les variétés, il existe une différence significative $(\mathrm{P}<0,0001)$ pour les deux conditions climatiques.

\section{DISCUSSION}

Les valeurs mesurées des différents paramètres ont varié d'une variété à une autre.
Pour la longueur du tubercule, les valeurs obtenues dans les deux conditions climatiques se rapprochent de celles obtenues par Wilson (1977) sur la patate douce. Cet auteur avait trouvé des valeurs qui ont varié entre $7,4 \mathrm{~cm}$ et $15,6 \mathrm{~cm}$. Par contre, pour le même paramètre, les valeurs obtenues par Tarini et al. (2007) sur d'autres variétés de patate douce cultivées au Burkina Faso ont varié entre $10 \mathrm{~cm}$ et $38,2 \mathrm{~cm}$. Nos résultats ont montré des diamètres du tubercule sensiblement comparables à ceux obtenus par Tarini et al. (2007) sur des variétés de patate douce cultivée au Burkina Faso soit de 3,36 à $8,45 \mathrm{Cm}$ et ceux obtenus par wilson en 1977 soit 3 à $5,3 \mathrm{~cm}$. Les résultats ont dû être influencés par la pluviométrie de la période de l'expérimentation $(227,6 \mathrm{~mm})$ très faible par rapport à la hauteur annuelle moyenne de pluie dans la zone de Ouagadougou qui se situe autour de $700 \mathrm{~mm}$ et $(178,49 \mathrm{~mm})$ aussi très faible par rapport à la hauteur annuelle moyenne de pluie dans la zone de Bongor qui oscille entre 800 et $900 \mathrm{~mm}$.

Le nombre du tubercule par plant a varié en fonction de la variété et l'environnement dans lequel, l'expérimentation a été menée. Dans tous les cas, les résultats obtenus sont sensiblement identiques à ceux obtenues chez les 4 variétés de patate douce à savoir Mugandé, Spk013, Kemb 10 et SPK004 cultivées à l'Ouest du Kenya, dont les valeurs étaient respectivement 4,$2 ; 3,1 ; 3,5$ et 2,8 (Ndolo et al., 2001). Toutefois, chez les variétés Yan Shu1 et White Delite cultivées à l'Est du Congo, le nombre de tubercules par plant est plus important, soit respectivement 15,5 et 14,0 tubercules par plant (Phemba et al., 1998). Ce nombre important obtenu chez ces variétés cultivées au Congo peut être lié au type du sol, la température du milieu et surtout la pluviométrie. Dans notre cas, la pluviométrie a été assez faible au moment de l'expérimentation. Tandis que, chez la lignée qualifiée de très productive (TIS 2328/6), le nombre moyen du tubercule est 8,5 Wilson (1977).

Les rendements en tubercule ont varié entre les variétés et aussi selon le milieu. 
Parmi toutes les variétés étudiées, la variété BF 108 a donné un rendement en tubercules très satisfaisant avec une matière sèche de la biomasse aérienne plus importante. Certains auteurs parmi lesquels Sajjapongse et Roan (1982) ont montré une corrélation entre le rendement et la matière sèche de la biomasse aérienne. Selon Sajjapongse et Roan (1982) une plante feuillue à tiges vigoureuses a très souvent une production de racine élevée. En outre, Austin et al. (1970) ont pensé aussi que la formation des racines est fonction de l'accumulation des photosynthétats, en particulier d'hydrates de carbone provenant de la partie aérienne. Cela montre que plus la plante de patate douce a un feuillage important, plus elle accumule des photosynthétats nécessaires pour la formation des racines. Le rendement de la variété $\mathrm{BF}$ 108 est supérieur à ceux obtenus chez les autres variétés. Mais à l'exception de la variété BF 140 les valeurs obtenues se rapprochent de celles obtenues chez des clones cultivés au Sénégal dont les valeurs variaient de 18 à 27,7 t/ha (clone $2(27,7$ t/ha), clone 27 (26,8 t/ha), clone 80-24 (24 t/ha), la variété locale (22 t/ha), walo (21,1 t/ha), Ndargu (18 t/ha) et le clone 29 (18,5 t/ha)) Huat (1999). Cependant, le rendement de la lignée la plus productive (TIS 2328/6) était de 68,1t/ha Wilson (1977).

Par ailleurs, les rendements en tubercule de la patate douce peuvent aussi être affectés en fonction de la saison au cours de laquelle la culture a été effectuée Zara et al. (1982). Pendant nos expérimentations, nous avons constaté que les résultats en termes de rendement sont importants en saison des pluies qu'en saison sèche malgré la faible pluviométrie relevée pendant ces expérimentations. Cependant, plusieurs études ont montré l'action de la température sur la répartition des assimilats et sur le développement des racines de réserve Kim (1961) et Sakr (1943). Kim (1961) a montré également que les plants de patate douce produisaient un plus grand nombre de racines de réserve, d'un poids accru quand la température de l'air était de $20^{\circ} \mathrm{c}$ pendant la période d'obscurité et de $29^{\circ} \mathrm{c}$ pendant tout le cycle. Mais les conditions pluviométriques n'ont pas été suffisantes pour donner un rendement très satisfaisant.

Sekioka (1970) a montré que les plants de patate douce produisent de quantités plus élevées en racine de stockage lorsqu'ils étaient soumis à une température du sol constante de $30{ }^{\circ} \mathrm{C}$, combiné à une température de l'air de $25{ }^{\circ} \mathrm{C}$ pendant la nuit. Pendant nos expérimentations, les températures moyennes relevées étaient 28,1 ${ }^{\circ} \mathrm{C}$ et $33,1{ }^{\circ} \mathrm{C}$ respectivement à Ouagadougou et à Bongor. En outre, la compacité du sol peut également avoir une influence significative sur le rendement Sajjepongse et Roan (1982). Le sol argileux est compact, il empêche donc le développement des racines tandis que le sol sablonneux le favorise.

La teneur en matière sèche variait d'une variété à une autre. La valeur la plus élevée est observée chez la variété BF 140 dans les deux conditions environnementales. Cette valeur n'est pas loin des valeurs obtenues chez les variétés Benikomachi et Caroline Lee $(41 \%$ et $39 \%)$ cultivées à l'Est du Congo. Ces variétés très appréciées pour leurs qualités organoleptiques, se sont caractérisées par un taux de matière sèche élevé Feruzi et al. (2001). Par contre, les variétés Yanshu 1 et White Delite cultivées aussi à 1'Est du Congo moins appréciées pour ces qualités organoleptiques, ont un taux de matière sèche de l'ordre de $26 \%$ à $28 \%$ Feruzi et al. (2001). Cette teneur en matière sèche importante chez les variétés BF 139 et BF 140 peut se justifier par le fait que leurs tubercules respectifs ont un taux d'humidité relativement faible. Pour les expérimentations menées, la différence en termes de matière sèche n'est pas grande entre les variétés utilisées.

Le rapport diamètre/longueur du tubercule donne une idée sur la forme du tubercule de la patate douce considérée. Les valeurs du rapport se rapprochant de 0,5 permettent d'identifier les tubercules ronds Tarini et al. (2007). Dans tous les cas, ce 
rapport est plus important chez les variétés $\mathrm{BF}$ 108 et TIB440060.

Le poids moyen du tubercule a varié aussi suivant les variétés. La variété ayant des tubercules de poids moyen le plus élevé est la BF 108 et le poids le plus faible est noté chez la variété $\mathrm{BF} 140$. A l'exception des valeurs obtenues chez la variété BF 108 pour les expérimentations menées en saison des pluies dans les deux conditions climatiques, certaines des valeurs sont très faibles car elles sont loin des valeurs obtenues chez les variétés Zapallo.420027 (500 g), Saliboro 187017-1 (666 g), Excel 440016 (200 g), TIB440060 (458 g) et Jewel $566638(240 \mathrm{~g})$ cultivées au Burkina Faso Tarini et al. (2007). Austin et Aung (1973) ont établi une corrélation entre le poids moyen du tubercule et la matière sèche de la biomasse aérienne. Les analyses statistiques ont montré également cette corrélation dans le cas de nos études.

\section{Conclusion}

Les paramètres agromorphologiques de dix (10) variétés de la patate douce (Ipomoea batatas (L.)Lam expérimentées dans les deux (2) conditions ont été évalués. Les résultats obtenus ont montré que la variété BF 108 est la plus productive en tubercules dans les deux conditions climatiques. Le poids moyen du tubercule, la longueur et le diamètre du tubercule, le rapport diamètre/longueur du tubercule et la matière sèche de la biomasse aérienne ont été aussi important chez la variété $\mathrm{BF} 108$ et dans les deux milieux. Les variétés BF 11, BF 59 et BF 108 se sont distinguées des autres par le nombre du tubercule par plant à Ouagadougou et la BF 108, BF 40, BF 11, BF 13 et Jewel à Bongor. En fin, la variété BF 140 a une teneur plus élevée en matière sèche du tubercule dans les deux (2) environnements. Vu la performance de la variété BF 108 à Bongor, nous pensons qu'elle peut très bien aussi s'adapter dans les autres zones agro écologiques du Tchad. Ainsi en termes de sélection, elle est bien indiquée.

\section{REMERCIEMENTS}

Nous tenons à remercier la Coopération française, l'Institut National de l'Environnement et de la Recherche Agricole (INERA) de Kamboinsé, l'Université de Ouagadougou et l'Ecole Normale Supérieure de Bongor (ENSB) qui ont soutenu financièrement, matériellement et moralement ce travail.

\section{REFERENCES}

Afuape SO, Nwankwo IIM, Omodamiro RM, Echendu TNC, Touré A. 2014. Studies on some important consumer and processing traits for breeding sweetpotato for varied End-uses. Am. J. Exp. Agr., 4(1): 114-124.

Austin ME, Aung LH, Graves B. 1970. Some observations on growth and development of sweet potato (Ipomoea batatas). J. Hort. Sci., 45(1):257-264.

Austin ME, Aung LH. 1973. Patterns of dry matter distribution during developement of sweet potato (Ipomoea batatas). $J$. Hort. Sci., 48(2): 257-264.

Bell A, Müch O, Schuler B. 2000. Les richesses du sol, les plantes à racines et tubercules en afrique : une contribution au développement des technologies de récoltes et après récoltes, DES : Feldafing

Feruzi M, Phemba P, Ngongo M, Hagenimana V, Lutaladio NB. 2001. Evaluation post récolte de six Génotypes de patate douce sélectionnés à l'Est du Congo. Afr. Crop. Sci. J., 9(1): 33-39.

Tarini A, Somda JC, Vebamba O, Yameogo KM, Belem J. 2007. Amélioration des apports en Vitamine des femmes et des enfants par la production et la consommation des patates douces à chair orange au Burkina Faso HKI, Ouagadougou.

Huat C, Mateille T, Huat J. 1999. Etude de la Sensibilité de la Patate Douce (Ipomoea batatas) aux Nématodes Phytoparasites du Genre Meloidogyne au Sénégal. IRD, PSI : Saint-Louis. 
Huang PC. 1982. La valeur nutritive de la patate douce. In La Patate Douce. CTA / ACCT. Shanhua; 45-54.

Kim YC 1961. Effect of thermoperiodism on tuber formation in Ipomoea batatas under controlled conditions. Pl. Physiol., 36(5): 680-684.

Mbanaso EO, Agwu AE, Anyanwu AC, Asumugha GN. 2012. Assessment of the exten of adoption of sweetpotato production Technology by farmers in the southeast agro-ecolgical zone of Nigeria. J. Agr. Soc. Res., 12(1): 124-136.

Ndolo PJ, Mcharo T, Carey EE, Gichuki ST, Ndinya C, Maling'a J. 2001. Participatory on-Farm selection of sweetpotato varities in western Kenya. Afr. Crop. Sci. J., 9(1) : 41-48.

Phemba P, Mutombo T, Lutaladio NB, Carey EE. 1998. Performance et stabilité de rendement des génotypes de patate douce dans divers environnements à l'Est du Congo. Afr. Crop. Sci. J., 6(2) :109-118.

Sajjapongse A, Roan YC. 1982. Les facteurs physiques affectant le rendement en racine de la patate douce. In Patate Douce. CTA / ACCT. Shanhua; 217229.
Sakr ESM. 1943. Effect of temperature on yield of the sweet potato. Am. Soc. Hort. Sci.Proc., 42(1): 517-518.

Sekioka. 1970. The effect of temperature on the translocation and accumulation of carbohydrate in sweet potato. Int symp. Trop. Root. Tub Crops., 2(1) : 37-40.

Sihachakr D, Cavalcante-Alves J.M, Tizroutine S, Allot M., Mussio I, Servaes A, Nzoghe D, Ducreux G. 1994. Embryogenèse somatique chez la patate douce (Ipomoea batatas (L.) Lam.) : Caractérisation et régénération des plantes. In Quel Avenir pour l'Amélioration des Plantes? AupelfUref (ed) John Libbey Eurotext. Paris ; 251-261.

Wilson LA. 1977. Tubérisation chez la patate douce (Ipomoea batatas L) Lam. In Patate Douce. CTA / ACCT (ed).Shanhua ; 89-104.

Zara DL, Guevas SE, Carlos JT. 1982. Le comportement des variétés de patate douce sous cocotiers. In Patate Douce, CTA / ACCT : Shanhua ; 251-259. 